\title{
Hopf bifurcation analysis in a stage-structure predator-prey model with two time delay
}

\author{
Bochen Han*, Shengming Yang, and Guangping Zeng \\ School of Mathematics and Physics, University of Science and Technology Beijing, Beijing, 100083, \\ PR China
}

\begin{abstract}
In this paper, we consider a predator-prey system with two time delays, which describes a prey-predator model with parental care for predators. The local stability of the positive equilibrium is analysed. By choosing the two time delays as the bifurcation parameter, the existence of Hopf bifurcation is studied. Numerical simulations show the positive equilibrium loses its stability via the Hopf bifurcation when the time delay increases beyond a threshold.
\end{abstract}

Keywords: Predator-prey model, Delay, Hopf bifurcation, Local stability.

\section{Introduction}

Predation relationship is one of the basic relationships of interactions among species that exist universally in nature, and it is also a major topic of population dynamics research. Partial differential system is an important tool to describe population ecology, in which the predator-prey model is one of the important models [1,15].

In the study of the predator-prey model, there are many factors that affect population dynamics, and it is impossible that every predator has the same ability to capture prey in reality. In nature, they all go through two stages of immaturity and maturity. Immature predators are raised by their parents. Therefore, the single-species growth model consisting of immature stage and mature stage has also received extensive attention in recent years [2$4,12-14]$. Nutrient availability, whether from their parents or from prey, can enrich the model [5].

At the same time, with the deepening of research, people found that the predator-prey model could not accurately describe the time lag behavior of some research objects, that is, the time lag phenomenon. In the process of population development, the population development is not only related to the current state, but also related to the previous period of time due to the influence of factors such as pregnancy, regeneration, incubation and maturity of the population [8-11]. At this time, it is very important to add time delay factor into the population model. Therefore, this paper adopts a stage structure predator-prey model with parental care with time delay.

The main content of this paper is as follows. In the Section II, we give the model and analyze the model without time delay. In the section III, we discuss the stability of the

*Corresponding author: 41821232@xs.ustb.edu.cn 
equilibrium point and the Hopf bifurcation, considering various cases with added time delays. Section IV uses numerical simulation to verify the theoretical results according to the parameter conditions. Concluding remarks are contained in section $\mathrm{V}$.

\section{Existence and stability of equilibria}

In this paper, we consider the stage-structure predator-prey model [6] with parental care

$$
\left\{\begin{array}{l}
\frac{d u(t)}{d t}=u(t)(r-\alpha u(t))-\beta u(t) v_{2}(t) \\
\frac{d v_{1}(t)}{d t}=k_{1} \beta u\left(t-\tau_{1}\right) v_{2}(t) \frac{v_{2}(t)}{\omega v_{1}(t)+v_{2}(t)}-m_{1} v_{1}(t)-k_{2} \beta u(t) v_{2}(t) \frac{\omega v_{1}(t)}{\omega v_{1}(t)+v_{2}(t)} \\
\frac{d v_{2}(t)}{d t}=k_{2} \beta u(t) v_{2}(t) \frac{\omega v_{1}\left(t-\tau_{2}\right)}{\omega v_{1}\left(t-\tau_{2}\right)+v_{2}(t)}-m_{2} v_{2}(t)
\end{array}\right.
$$

where $\mathrm{u}, v_{1}$ and $v_{2}$ denote the densities of prey, immature predator and mature predator, respectively.r is the intrinsic grow rate of the prey. $\alpha$ is the density dependent coefficient of the prey. $\beta$ is the attacking rate of the mature predator at the prey. $\omega$ measures the relative consumption ratio between one immature predator and one mature predator. $k_{1}$ is a conversion coefficient of the immature predator. $k_{2}$ is a proportional constant and this kind of transition rate was used by Roughgarden et al.[7] for an open marine population. $m_{1}$ and $m_{2}$ are the death of immature predator and mature predator. We always assume that adult predators forage the prey and provide parental care to their offspring and all the parameters in system (1.1) are assumed to be positive.

Using the following parameters transformation:

the model (2.1) becomes

$$
\begin{aligned}
k_{2} \beta u / m_{2} \rightarrow u, w v_{1} & \rightarrow v_{1}, v_{2} \rightarrow v_{2}, m_{2} t \rightarrow t \\
k_{2} \beta u / m_{2} \rightarrow u, w v_{1} & \rightarrow v_{1}, v_{2} \rightarrow v_{2}, m_{2} t \rightarrow t
\end{aligned}
$$

$$
\left\{\begin{array}{l}
\frac{\mathrm{d} u}{\mathrm{~d} t}=u\left(a-b u-c v_{2}\right) \\
\frac{\mathrm{d} v_{1}}{\mathrm{~d} t}=\frac{k u\left(t-\tau_{1}\right) v_{2}^{2}}{v_{1}+v_{2}}-m v_{1}-\frac{\omega u v_{1} v_{2}}{v_{1}+v_{2}} \\
\frac{\mathrm{d} v_{2}}{\mathrm{~d} t}=\frac{u v_{1}\left(t-\tau_{2}\right) v_{2}}{v_{1}\left(t-\tau_{2}\right)+v_{2}}-v_{2}
\end{array}\right.
$$

where $a=r / m, b=\alpha /\left(k_{2} \beta\right), c=\beta / m_{2}, k=w k_{1} / k_{2}, m=m_{1} / m_{2}$.

The model (2.1) has a unique positive equilibrium if and only if

$$
a>b, k(a-b)^{2}>b[\omega(a-b)+b m]
$$

We denote the unique positive equilibrium as $\tilde{u}=\left(u^{*}, v_{1}^{*}, v_{2}^{*}\right)$, where

$$
\begin{aligned}
& \left\{\begin{array}{l}
u^{*}=\frac{a+c v_{1}^{*}}{b+c v_{1}^{*}} \\
v_{1}^{*}=\frac{-[2 b m+\omega(a-b)]+\left\{[2 b m+\omega(a-b)]^{2}-4 m\left\{b[b m+\omega(a-b)]-k(a-b)^{2}\right\}\right\}^{\frac{1}{2}}}{2 m c} \\
v_{2}^{*}=\frac{(a-b) v_{1}^{*}}{b+c v_{1}^{*}} \\
J=\left(\begin{array}{ccc}
-b u^{*} & 0 & -c u^{*} \\
\frac{v_{2}^{*}\left(k v_{2}^{*}-\omega v_{1}^{*}\right)}{v_{1}^{*}+v_{2}^{*}} & -\frac{(k+\omega) u^{*} v_{2}^{*}+m\left(v_{1}^{*}+v_{2}^{*}\right)^{2}}{\left(v_{1}^{*}+v_{2}^{*}\right)^{2}} & \frac{u^{*}\left(2 k v_{1}^{*} v_{2}^{*}+k v_{2}^{* 2}-\omega v_{1}^{* 2}\right)}{\left(v_{1}^{*}+v_{2}^{*}\right)^{2}} \\
\frac{v_{1}^{*} v_{2}^{*}}{v_{1}^{*}+v_{2}^{*}} & \frac{u^{*} v_{2}^{* 2}}{\left(v_{1}^{*}+v_{2}^{*}\right)^{2}} & -\frac{u^{*} v_{1}^{*} v_{2}^{*}}{\left(v_{1}^{*}+v_{2}^{*}\right)^{2}}
\end{array}\right)
\end{array}\right.
\end{aligned}
$$

The characteristic equation of $J: \operatorname{det}\left(J-\lambda I_{3}\right)=0$, marked as: $\Delta(\lambda, 0)$. 
$\Delta(\lambda, 0)$

$$
\begin{gathered}
=\left|\begin{array}{ccc}
-b u^{*}-\lambda & 0 & -c u^{*} \\
\frac{v_{2}^{*}\left(k v_{2}^{*}-\omega v_{1}^{*}\right)}{v_{1}^{*}+v_{2}^{*}} & -\frac{(k+\omega) u^{*} v_{2}^{*}+m\left(v_{1}^{*}+v_{2}^{*}\right)^{2}}{\left(v_{1}^{*}+v_{2}^{*}\right)^{2}}-\lambda & \frac{u^{*}\left(2 k v_{1}^{*} v_{2}^{*}+k v_{2}^{* 2}-\omega v_{1}^{* 2}\right)}{\left(v_{1}^{*}+v_{2}^{*}\right)^{2}} \\
\frac{v_{1}^{*} v_{2}^{*}}{v_{1}^{*}+v_{2}^{*}} & \frac{u^{*} v_{2}^{* 2}}{\left(v_{1}^{*}+v_{2}^{*}\right)^{2}} & -\frac{u^{*} v_{1}^{*} v_{2}^{*}}{\left(v_{1}^{*}+v_{2}^{*}\right)^{2}}-\lambda
\end{array}\right| \\
J=\left(\begin{array}{lll}
a_{11} & a_{12} & a_{13} \\
a_{21} & a_{22} & a_{23} \\
a_{31} & a_{32} & a_{33}
\end{array}\right)
\end{gathered}
$$

Then the characteristic equation is simplified as:

$$
\begin{gathered}
\lambda^{3}-\left(a_{11}+a_{22}+a_{33}\right) \lambda^{2}-\left(a_{13} a_{31}+a_{23} a_{32}-a_{11} a_{22}-a_{11} a_{33}-a_{22} a_{33}\right) \lambda \\
-a_{11} a_{22} a_{33}+a_{11} a_{23} a_{32}-a_{13} a_{21} a_{32}+a_{13} a_{22} a_{31}=0
\end{gathered}
$$

Then

$$
\begin{aligned}
& \Delta 1=a_{1}=-\left(a_{11}+a_{22}+a_{33}\right)>0 \\
& \Delta 2=\left(\begin{array}{ll}
a_{1} & a_{0} \\
a_{3} & a_{2}
\end{array}\right)=a_{1} a_{2}-a_{3} a_{0}=\left(a_{11}+a_{22}+a_{33}\right)\left(a_{13} a_{31}+a_{23} a_{32}-\right. \\
&\left.a_{11} a_{22}-a_{11} a_{33}-a_{22} a_{33}\right)-\left(-a_{11} a_{22} a_{33}+a_{11} a_{23} a_{32}-\right. \\
& \Delta 3=\left|\begin{array}{lll}
a_{1} & \left.a_{13} a_{21} a_{32}+a_{13} a_{22} a_{31}\right)>0 \\
a_{3} & a_{2} & a_{1} \\
a_{5} & a_{4} & a_{3}
\end{array}\right|=\Delta 2 \times a_{3}>0
\end{aligned}
$$

So $a_{1}>0, a_{3}>0, a_{1} a_{2}-a_{3}>0$.

The following result was given in paper [6].

Lemma 2.1. If $m<1$, then the positive constant equilibrium $\tilde{u}=\left(u^{*}, v_{1}^{*}, v_{2}^{*}\right)$ of $(2.1)$ is locally asymptotically stable.

\section{Stability and Hopf bifurcation}

In this section, we will discuss existence of a Hopf bifurcation at the positive equilibrium in three cases.

Case 1. $\tau_{1} \neq 0, \tau_{2}=0$

The linearization of system around the equilibrium $\tilde{u}$ can be expressed by

where:

$$
\frac{\mathrm{d} \tilde{u}}{\mathrm{~d} t}=J_{1} u^{*}+J_{2} u^{*}(\tau)
$$

$$
\begin{aligned}
& J_{1}=\left(\begin{array}{ccc}
-b u^{*} & 0 & -c u^{*} \\
0 & -\frac{(k+\omega) u^{*} v_{2}^{*}+m\left(v_{1}^{*}+v_{2}^{*}\right)^{2}}{\left(v_{1}^{*}+v_{2}^{*}\right)^{2}} & \frac{u^{*}\left(2 k v_{1}^{*} v_{2}^{*}+k v_{2}^{* 2}-\omega v_{1}^{* 2}\right)}{\left(v_{1}^{*}+v_{2}^{*}\right)^{2}} \\
\frac{v_{1}^{*} v_{2}^{*}}{v_{1}^{*}+v_{2}^{*}} & \frac{u^{*} v_{2}^{* 2}}{\left(v_{1}^{*}+v_{2}^{*}\right)^{2}} & -\frac{u^{*} v_{1}^{*} v_{2}^{*}}{\left(v_{1}^{*}+v_{2}^{*}\right)^{2}}
\end{array}\right) \\
& J_{2}=\left(\begin{array}{ccc}
0 & 0 & 0 \\
\frac{v_{2}^{*}\left(k v_{2}^{*}-\omega v_{1}^{*}\right)}{v_{1}^{*}+v_{2}^{*}} & 0 & 0 \\
0 & 0 & 0
\end{array}\right) \\
& J=\left(\begin{array}{ccc}
-b u^{*} & 0 & -c u^{*} \\
\frac{v_{2}^{*}\left(k v_{2}^{*}-\omega v_{1}^{*}\right)}{v_{1}^{*}+v_{2}^{*}} e^{-\lambda \tau} & \frac{(k+\omega) u^{*} v_{2}^{*}+m\left(v_{1}^{*}+v_{2}^{*}\right)^{2}}{\left(v_{1}^{*}+v_{2}^{*}\right)^{2}} & \frac{u^{*}\left(2 k v_{1}^{*} v_{2}^{*}+k v_{2}^{* 2}-\omega v_{1}^{* 2}\right)}{\left(v_{1}^{*}+v_{2}^{*}\right)^{2}} \\
\frac{v_{1}^{*} v_{2}^{*}}{v_{1}^{*}+v_{2}^{*}} & \frac{u^{*} v_{2}^{* 2}}{\left(v_{1}^{*}+v_{2}^{*}\right)^{2}} & -\frac{u^{*} v_{1}^{*} v_{2}^{*}}{\left(v_{1}^{*}+v_{2}^{*}\right)^{2}}
\end{array}\right)
\end{aligned}
$$




$$
J=\left(\begin{array}{lll}
a_{11} & a_{12} & a_{13} \\
a_{21} & a_{22} & a_{23} \\
a_{31} & a_{32} & a_{33}
\end{array}\right)
$$

Then the associated characteristic equation of (3.1) is

where:

$$
\lambda^{3}+B_{1} \lambda^{2}+B_{2} \lambda+B_{3}+B_{4} \mathrm{e}^{-\lambda \tau}=0
$$

$B_{1}=-a_{11}-a_{22}-a_{33}$

$B_{2}=a_{11} * a_{22}+a_{11} * a_{33}-a_{13} * a_{31}+a_{22} * a_{33}-a_{23} * a_{32}$

$B_{3}=-a_{11} * a_{22} * a_{33}+a_{11} * a_{23} * a_{32}+a_{13} * a_{22} * a_{31}$

$B_{4}=-a_{13} * a_{21} * a_{32}$

For $\tau>0$, if $\lambda=\mathrm{i} \omega(\omega>0)$ is a root of (3.2), then we have

$$
\left\{\begin{array}{l}
\omega^{3}-B_{2} \omega+B_{4} \sin \omega \tau=0 \\
B_{1} \omega^{2}-B_{3}-B_{4} \cos \omega \tau=0
\end{array}\right.
$$

Adding up the squares of both equations, we obtain

$$
\omega^{6}+\left(B_{1}^{2}-2 B_{2}\right) \omega^{4}+\left(B_{2}^{2}-2 B_{1} B_{3}\right) \omega^{2}+B_{3}^{2}-B_{4}^{2}=0
$$

Denote $z=\omega^{2}$, then (3.4) becomes

where $p=B_{1}^{2}-2 B_{2}, q=B_{2}^{2}-2 B_{1} B_{3}, r=B_{3}^{2}-B_{4}^{2}$

$$
z^{3}+p z^{2}+q z+r=0
$$

Lemma 3.1. if $B_{3}^{2}-B_{4}^{2}>0$, then equation (3.5) has at least one positive real root.

Suppose the equation (3.5) has at least one positive root, which is general, and the assumption (3.5) has three positive roots, which are recorded as $z_{1}, z_{2}, z_{3}$.

The equation (3.5) also has three positive roots

$\omega_{k}=\sqrt{v_{k}} \quad k=1,2,3$

$\omega=\omega_{j}$, the root of order (3.4) could be obtained:

where:

$$
\tau_{j}^{(n)}=\frac{\arccos \left(h\left(\omega_{j}\right)\right)}{\omega_{j}}+\frac{2 n \pi}{\omega_{j}}, n=1,2, \ldots
$$

$$
h\left(\omega_{j}\right)=\frac{B_{1} \omega_{j}^{2}-B_{3}}{B_{4}}, \tau^{*}=\tau_{j_{0}}^{(0)}=\min _{j=1,2,3}\left\{\tau_{j}^{(0)}\right\}, \omega^{*}=\omega_{j_{0}}
$$

Lemma 3.2. Suppose $h^{\prime}(z) \neq 0$. If $\tau=\tau^{*}$, then $\frac{\mathrm{d}(\operatorname{Re} \lambda)}{\mathrm{d} \tau} \neq 0$

Proof. Sorting equations (2.11) can be obtained

Differentiating the above equations with respect to $\tau$, we have

$$
\mathrm{e}^{-\lambda t}=\frac{-\lambda^{3}-B_{1} \lambda^{2}-B_{2} \lambda-B_{3}}{B_{4}}
$$

$$
\lambda B_{4} \mathrm{e}^{-\lambda \tau}=\left(3 \lambda^{2}+2 B_{1} \lambda+B_{2}-B_{4} \tau \mathrm{e}^{-\lambda \tau}\right) \frac{\mathrm{d} \lambda}{\mathrm{d} \tau}
$$

Sort it out

$$
\left(\frac{\mathrm{d} \lambda}{\mathrm{d} \tau}\right)^{-1}=\frac{3 \lambda^{2}+2 B_{1} \lambda+B_{2}}{B_{4} \lambda \mathrm{e}^{-\lambda \tau}}-\frac{\tau}{\lambda}
$$

From (3.7) and (3.9) we have:

$$
\left(\frac{\mathrm{d} \lambda}{\mathrm{d} \tau}\right)^{-1}=\frac{3 \lambda^{2}+2 B_{1} \lambda+B_{2}}{\lambda\left(-\lambda^{3}-B_{1} \lambda^{2}-B_{2} \lambda-B_{3}\right)}-\frac{\tau}{\lambda}
$$

By substituting $\lambda=\omega i, \omega=\omega^{*}$

$$
\operatorname{Re}\left\{\left(\frac{\mathrm{d} \lambda}{\mathrm{d} \tau}\right)^{-1}\right\}=\frac{3 \omega^{6}+\left(2 B_{1}^{2}-4 B_{2}\right) \omega^{4}+\left(B_{2}^{2}-2 B_{1} B_{3}\right) \omega^{2}}{\omega^{8}+\left(B_{1}-2 B_{2}\right) \omega^{6}+\left(B_{2}^{2}-2 B_{1} B_{3}\right) \omega^{4}+B_{3}^{2} \omega^{2}}
$$

As $z^{*}=\omega^{*}$ and $h^{\prime}(z) \neq 0$,we have 
This completes the Proof.

$$
\operatorname{Re}\left\{\left(\frac{\mathrm{d} \lambda}{\mathrm{d} \tau}\right)^{-1}\right\}=\frac{\omega^{* 2} h^{\prime}\left(z^{*}\right)}{\omega^{* 2} h\left(z^{*}\right)+B_{4}^{2} \omega^{* 2}} \neq 0
$$

By the lemma 3.1 and 3.2, the transversality condition is satisfied. In addition the equation $\Delta(\mathrm{i} \omega, \tau)=0$ has a pair of simple purely imaginary roots $\pm \mathrm{i} \omega^{*}$ at $\tau^{*}$. Therefore we have the following theorem.

Theorem 3.3. Suppose that (2.3) holds, then we can obtain Hopf bifurcation of the system which holds at $\tau=\tau^{*}$

Case 2. $\tau_{1}=0, \tau_{2} \neq 0$

The proof is similar to the Case $\mathbf{1 .}$

Case 3. $\tau_{1}=\tau_{2} \neq 0$

The linearization of system (2.1) around the equilibrium $u^{*}$ can be expressed by

$$
\frac{\mathrm{d} x}{\mathrm{~d} t}=J_{1} x+J_{2} x(\tau)
$$

Where

$$
\begin{aligned}
& J_{1}=\left(\begin{array}{ccc}
-b u^{*} & 0 & -c u^{*} \\
0 & -\frac{(k+\omega) u^{*} v_{2}^{*}+m\left(v_{1}^{*}+v_{2}^{*}\right)^{2}}{\left(v_{1}^{*}+v_{2}^{*}\right)^{2}} & \frac{u^{*}\left(2 k v_{1}^{*} v_{2}^{*}+k v_{2}^{* 2}-\omega v_{1}^{* 2}\right)}{\left(v_{1}^{*}+v_{2}^{*}\right)^{2}} \\
0 & \frac{u^{*} v_{2}^{* 2}}{\left(v_{1}^{*}+v_{2}^{*}\right)^{2}} & -\frac{u^{*} v_{1}^{*} v_{2}^{*}}{\left(v_{1}^{*}+v_{2}^{*}\right)^{2}}
\end{array}\right) \\
& J_{2}=\left(\begin{array}{ccc}
0 & 0 & 0 \\
\frac{v_{2}^{*}\left(k v_{2}^{*}-\omega v_{1}^{*}\right)}{v_{1}^{*}+v_{2}^{*}} & 0 & 0 \\
\frac{v_{1}^{*} v_{2}^{*}}{v_{1}^{*}+v_{2}^{*}} & 0 & 0
\end{array}\right)
\end{aligned}
$$

The coefficient matrix of a linearized system can be expressed as:

$$
\begin{aligned}
J & =\left(\begin{array}{ccc}
-b u^{*} & 0 & -c u^{*} \\
\frac{v_{2}^{*}\left(k v_{2}^{*}-\omega v_{1}^{*}\right)}{v_{1}^{*}+v_{2}^{*}} e^{-\lambda \tau} & -\frac{(k+\omega) u^{*} v_{2}^{*}+m\left(v_{1}^{*}+v_{2}^{*}\right)^{2}}{\left(v_{1}^{*}+v_{2}^{*}\right)^{2}} & \frac{u^{*}\left(2 k v_{1}^{*} v_{2}^{*}+k v_{2}^{* 2}-\omega v_{1}^{* 2}\right)}{\left(v_{1}^{*}+v_{2}^{*}\right)^{2}} \\
\frac{v_{1}^{*} v_{2}^{*}}{v_{1}^{*}+v_{2}^{*}} & \frac{u^{*} v_{2}^{* 2}}{\left(v_{1}^{*}+v_{2}^{*}\right)^{2}} & -\frac{u^{*} v_{1}^{*} v_{2}^{*}}{\left(v_{1}^{*}+v_{2}^{*}\right)^{2}}
\end{array}\right) \\
J & =\left(\begin{array}{lll}
a_{11} & a_{12} & a_{13} \\
a_{21} & a_{22} & a_{23} \\
a_{31} & a_{32} & a_{33}
\end{array}\right)
\end{aligned}
$$

Then the associated characteristic equation of (3.13) is

$$
\lambda^{3}+B_{1} \lambda^{2}+\left(B_{2}+B_{4} e^{-\lambda \tau}\right) \lambda+B_{3}+B_{5} \mathrm{e}^{-\lambda \tau}
$$

where:

$$
\begin{aligned}
& B_{1}=-a_{11}-a_{22}-a_{33} \\
& B_{2}=a_{11} * a_{22}+a_{11} * a_{33}-a_{13} * a_{31}+a_{22} * a_{33}-a_{23} * a_{32} \\
& B_{3}=-a_{11} * a_{22} * a_{33}+a_{11} * a_{23} * a_{32}+a_{13} * a_{22} * a_{31} \\
& B_{4}=-a_{13} * a_{21} * a_{32}
\end{aligned}
$$

For $\tau>0$, if $\lambda=\mathrm{i} \omega(\omega>0)$ is a root of (3.14), then we have

Adding up the squares of both equations, we obtain:

$$
\left\{\begin{array}{l}
\omega^{3}-B_{2} \omega-B_{4} \cos \omega \tau+B_{5} \sin \omega \tau=0 \\
B_{1} \omega^{2}-B_{3}-B_{4} \sin \omega \tau-B_{5} \cos \omega \tau=0
\end{array}\right.
$$

$$
\omega^{6}+\left(B_{1}^{2}-2 B_{2}\right) \omega^{4}+\left(B_{2}^{2}-2 B_{1} B_{3}-B_{4}^{2}\right) \omega^{2}+B_{3}^{2}-B_{5}^{2}=0
$$

Denote $z=\omega^{2}$, then (3.16) becomes 
where $p=B_{1}^{2}-2 B_{2}, q=B_{2}^{2}-2 B_{1} B_{3}-B_{4}^{2}, r=B_{3}^{2}-B_{5}^{2}$

$$
z^{3}+p z^{2}+q z+r=0
$$

Suppose the equation (3.17) has at least one positive root, which is general, and the assumption (3.19) has three positive roots, which are recorded as $z_{1}, z_{2}, z_{3}$.

The equation (3.16) also has three positive roots

$\omega_{k}=\sqrt{v_{k}} \quad k=1,2,3$

$\omega=\omega_{j}$, the root of order (3.14) could be obtained:

where:

$$
\tau_{j}^{(n)}=\frac{\arccos \left(h\left(\omega_{j}\right)\right)}{\omega_{j}}+\frac{2 n \pi}{\omega_{j}}, n=1,2, \ldots
$$

$$
\begin{aligned}
& h\left(\omega_{j}\right)=\frac{B_{4} \omega_{j}^{4}+\left(B_{1} B_{5}-B_{2} B_{4}\right) \omega_{j}^{2}+B_{3} B_{5}}{B_{4}^{2} \omega_{j}^{2}+B_{5}^{2}} \\
& \tau^{*}=\tau_{j 0}^{(0)}=\min _{j=1,2,3}\left\{\tau_{j}^{(0)}\right\}, \omega^{*}=\omega_{j 0}
\end{aligned}
$$

Lemma 3.2 Suppose $h^{\prime}(z) \neq 0$.If $\tau=\tau^{*}$, then $\frac{\mathrm{d}(\operatorname{Re} \lambda)}{\mathrm{d} \tau} \neq 0$

Proof. Sorting equations (3.15) can be obtained

Differentiating the above equations with respect to $\tau$, we have

$$
\mathrm{e}^{-\lambda \tau}=\frac{-\lambda^{3}-B_{1} \lambda^{2}-B_{2} \lambda-B_{3}}{B_{4} \lambda+B_{5}}
$$

$$
\begin{array}{r}
\lambda^{2} B_{4} \mathrm{e}^{-\lambda \tau}+\lambda B_{5} \mathrm{e}^{-\lambda r}=\left(3 \lambda^{2}+2 B_{1} \lambda+B_{2}-B_{4} \lambda \tau \mathrm{e}^{-\lambda \tau}-B_{5} \lambda \mathrm{e}^{-\lambda \tau}\right) \frac{\mathrm{d} \lambda}{\mathrm{d} \tau} \\
\left(\frac{\mathrm{d} \lambda}{\mathrm{d} \tau}\right)^{-1}=\frac{3 \lambda^{2}+2 B_{1} \lambda+B_{2}+B_{4} \mathrm{e}^{-\lambda \tau}-B_{4} \lambda \tau \mathrm{e}^{-\lambda t}-B_{5} \tau \mathrm{e}^{-\lambda \tau}}{B_{4} \lambda^{2} \mathrm{e}^{-\lambda \tau}+B_{5} \lambda \mathrm{e}^{-\lambda t}} \\
=\frac{3 \lambda^{2}+2 B_{1} \lambda+B_{2}}{\lambda\left(B_{4} \lambda+B_{5}\right) \mathrm{e}^{-\lambda \tau}}+\frac{B_{4} \mathrm{e}^{-\lambda \tau}}{\left(B_{4} \lambda^{2}+B_{5} \lambda\right) \mathrm{e}^{-\lambda \tau}}-\frac{\tau\left(B_{4} \lambda+B_{5}\right) \mathrm{e}^{-\lambda t}}{\lambda\left(B_{4} \lambda+B_{5}\right) \mathrm{e}^{-\lambda t}}
\end{array}
$$

From (3.19) and (3.21) we have:

By substituting $\lambda=i \omega, \omega=\omega^{*}$

$$
\left(\frac{\mathrm{d} \lambda}{\mathrm{d} \tau}\right)^{-1}=\frac{3 \lambda^{2}+2 B_{1} \lambda+B_{2}}{-\lambda\left(\lambda^{3}+B_{1} \lambda^{2}+B_{2} \lambda+B_{3}\right)}+\frac{B_{4}}{B_{4} \lambda^{2}+B_{5} \lambda}-\frac{\tau}{\lambda}
$$

$\operatorname{Re}\left\{\left(\frac{\mathrm{d} \lambda}{\mathrm{d} \tau}\right)^{-1}\right\}=\frac{1}{\omega}\left(\frac{\left(B_{2}-3 \omega^{2}\right)\left(B_{2} \omega-\omega^{3}\right)}{\left(B_{2} \omega-\omega^{3}\right)^{2}+\left(B_{1} \omega^{2}-B_{3}\right)^{2}}-\frac{B_{4}^{2} \omega^{3}}{B_{4}^{2} \omega^{3}+B_{5}^{2} \omega^{2}}\right)$

$$
=\frac{1}{\omega^{2}}\left\{1+\frac{B_{4} \omega^{8}+B_{5}^{2} \omega^{6}+\left(2 B_{2} B_{5}^{2}+2 B_{1} B_{3} B_{4}\right) \omega^{4}}{\left[\left(B_{2} \omega-\omega^{3}\right)^{2}+\left(B_{1} \omega^{2}-B_{3}\right)^{2}\right]\left(B_{4}^{2} \omega^{2}+B_{5}^{2}\right)}-\frac{B_{2}^{2} B_{4}^{2} \omega^{4}+B_{3}^{2} B_{4}^{2} \omega^{2}+B_{3}^{2} B_{5}^{2}}{\left[\left(B_{2} \omega-\omega^{3}\right)^{2}+\left(B_{1} \omega^{2}-B_{3}\right)^{2}\right]\left(B_{4}^{2} \omega^{2}+B_{5}^{2}\right)}\right\}
$$

Obviously $1-\frac{B_{2}^{2} B_{4}^{2} \omega^{4}+B_{3}^{2} B_{4}^{2} \omega^{2}+B_{3}^{2} B_{5}^{2}}{\left[\left(B_{2} \omega-\omega^{3}\right)^{2}+\left(B_{1} \omega^{2}-B_{3}\right)^{2}\right]\left(B_{4}^{2} \omega^{2}+B_{5}^{2}\right)}>0$.

So the equation $\Delta(i \omega, \tau)=0$ has a pair of simple purely imaginary roots $\pm i \omega^{*}$ at $\tau^{*}$. So we can obtain Hopf bifurcation of the system which holds at $\tau=\tau^{*}$.

\section{Numerical simulation}

In this section, we carry out numerical simulation to demonstrate the analytical results. We use simple Euler method with a time step of $\Delta \mathrm{t}=0.01$.

Moreover, this set of parameters gives a unique positive equilibrium:

Of which

$$
\tilde{u}=\left(u^{*}, v_{1}^{*}, v_{2}^{*}\right)=(0.336,0.368,2.082)
$$

$$
a=45, b=\frac{5}{12}, c=20, k=\frac{4}{15}, m=1, \omega=0.8
$$



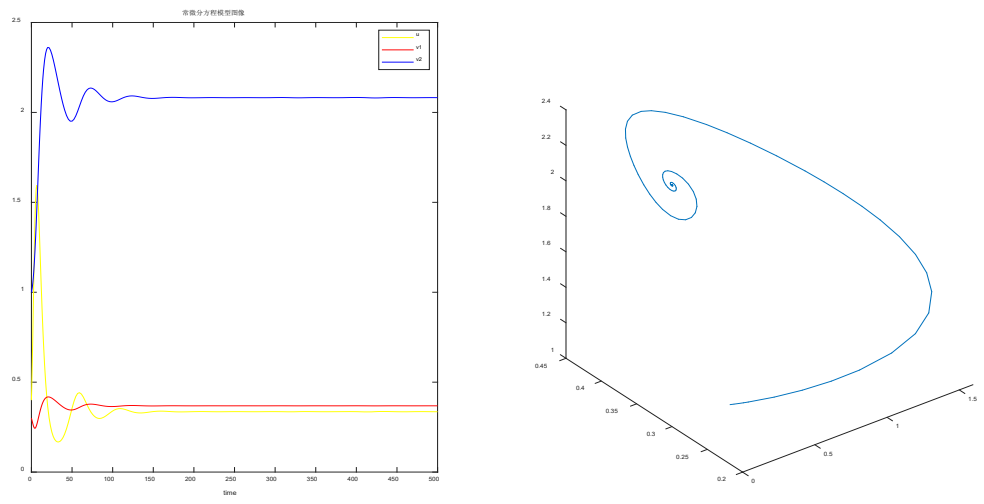

Fig. 6.1 Population dynamics (left) and phase portraits (right) for differential equation model.

Figure 6.1 shows the equilibrium is locally asymptotically stable (left), and the density phase diagram simulation of prey, immature predator and mature predator and the dynamic curve of their density over time (right).
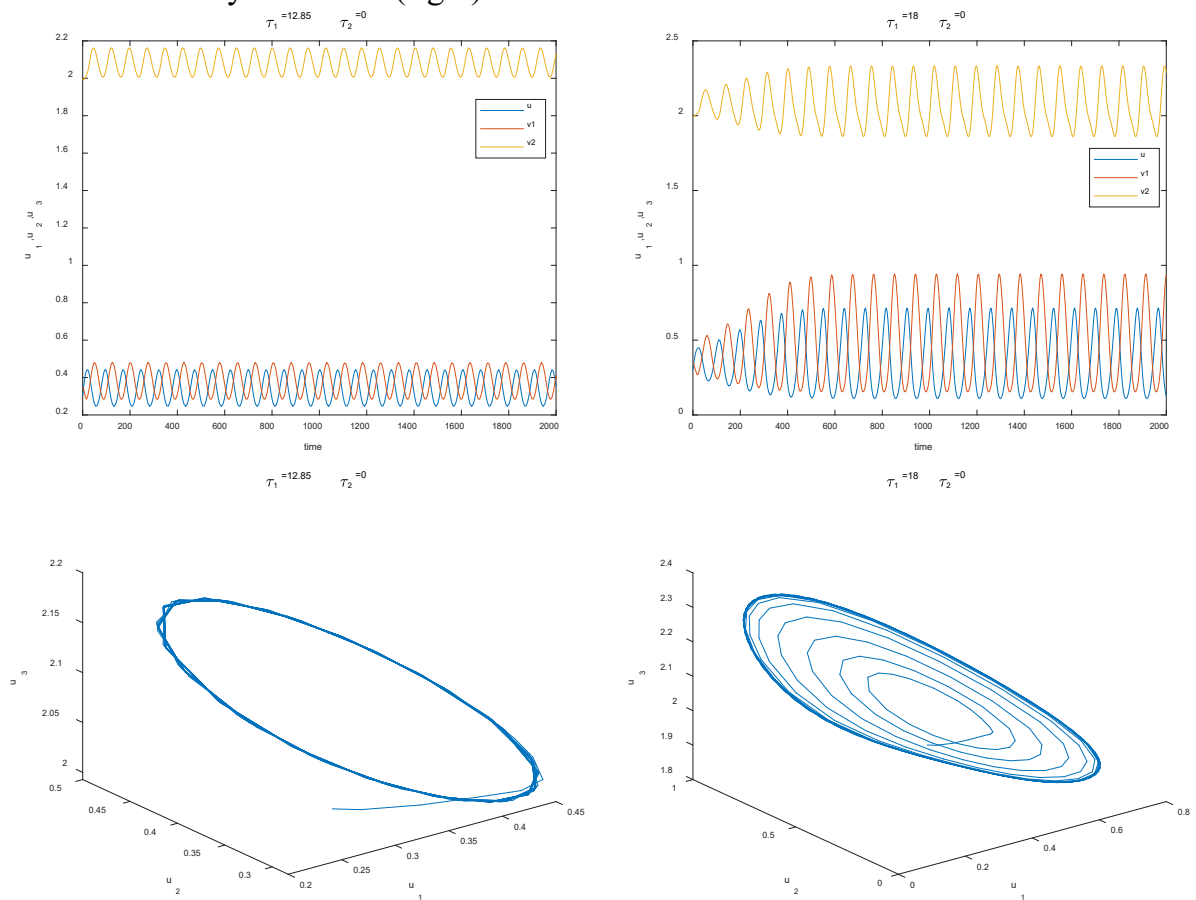

Fig. 6.2 Population dynamics (top) and phase portraits (bottom) for the temporal model at $\tau=12.85$ (left) and $\tau=18$ (right).

Figure 6.2 shows that in the case $\tau_{1} \neq 0, \tau_{2}=0$, the equilibrium is locally stable when $\tau<\tau^{*}(12.85)$, the limit cycle appears when $\tau=\tau^{*}$ and unstable when $\tau>\tau^{*}$. 

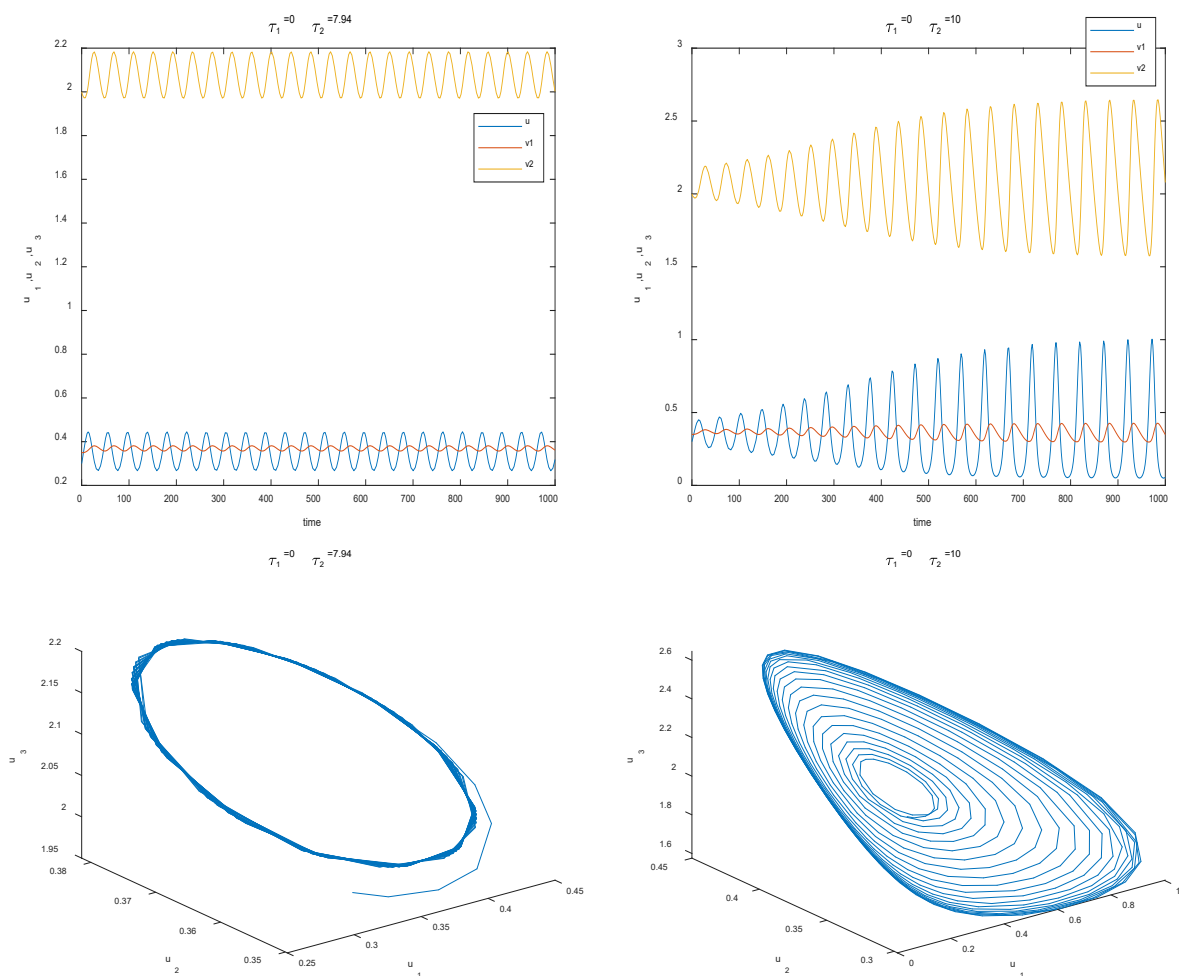

Fig. 6.3 Population dynamics (top) and phase portraits (bottom) for the temporal model at $\tau=7.94$ (left) and $\tau=10$ (right).

Figure 6.3 shows that in the case $\tau_{1}=0, \tau_{2} \neq 0$, the equilibrium is locally stable when $\tau<\tau^{*}$ (7.94), the limit cycle appears when $\tau=\tau^{*}$ and unstable when $\tau>\tau^{*}$.
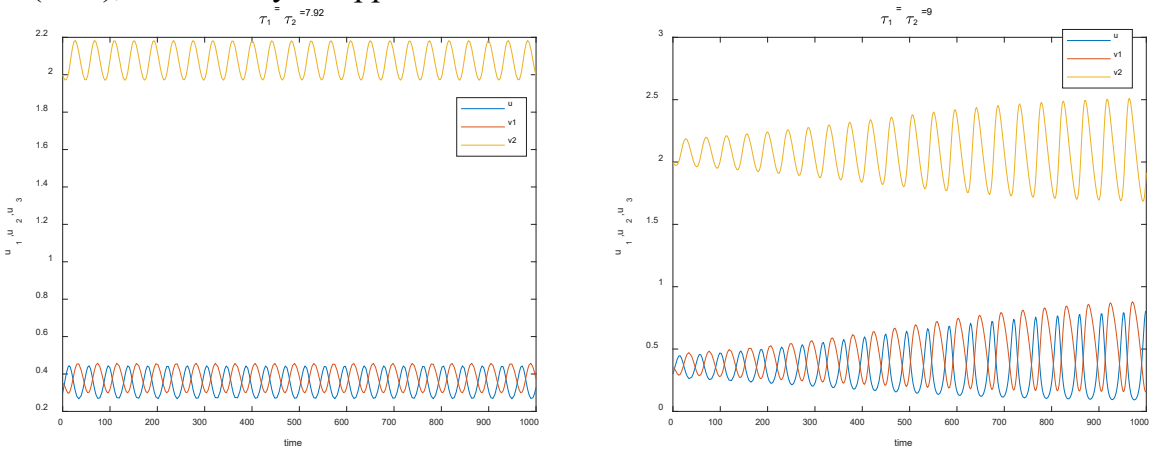

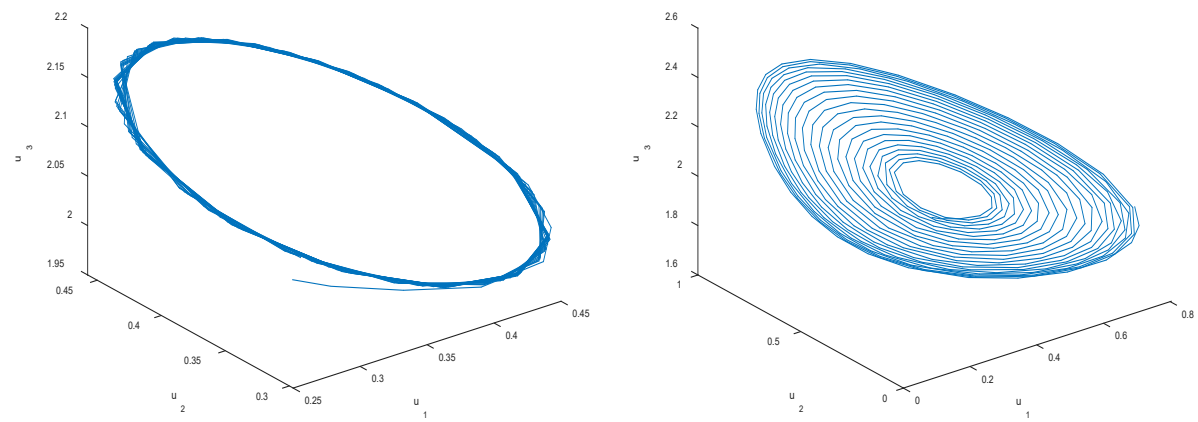

Fig. 6.4 Population dynamics (top) and phase portraits (bottom) for the temporal model at $\tau=7.92$ (left) and $\tau=9$ (right).

Figure 6.4 shows that in the case $\tau_{1}=\tau_{2} \neq 0$, the equilibrium is locally stable when $\tau<\tau^{*}$ (7.92), the limit cycle appears when $\tau=\tau^{*}$ and unstable when $\tau>\tau^{*}$.

\section{Conclusion}

In this paper, we study a strongly coupled nonlinear reaction diffusion system arising from a prey-predator model with parental care for predators. Sufficient condition which ensures the stability of equilibrium and the existence of Hopf bifurcation are obtained. Numerical simulations verify our results.

When $\tau_{1}=\tau_{2}=0$, we prove the local stability of the positive equilibrium applying Routh-Hurwitz criterion. Further, we study the existence of a Hopf bifurcation in three different cases $\tau_{1} \neq 0, \tau_{2}=0, \tau_{1}=0, \tau_{2} \neq 0$ and $\tau_{1}=\tau_{2} \neq 0$ at the positive equilibrium. In case one the threshold value of $\tau_{1}$ is 12.85 . In case two the threshold of $\tau_{2}$ is 7.94 . In case three the threshold is $\tau_{1}=\tau_{2}=7.94$. we only considered two equal delays. It would be more complicated if two delays have different value and deserve more study.

\section{References}

1. Xiao-Ke Sun, Hai-Feng Huo, Cao-Chuan Ma, Hopf bifurcation and stability in predator-prey model with a stage-structure for prey, Applied Mathematics and Computation. 219 (2013) 10313-10324.

2. M. Agarwal, S. Devi, Persistence in a ratio-dependent predator-prey-resource model with stage structure for prey, Int. J. Biomath. 3 (2010) 313-336.

3. W.G. Aiello, H.I. Freedman, A time-delay model of single-species growth with stage structure, Math. Biosci. 101 (1990) 139-153.

4. R. Shi, L. Chen, The study of ratio-dependent predator prey model with stage-structure in the prey, Nonlinear Dyn. 58 (2009) 443-451.

5. Jia Liu, Cross-diffusion induced stationary patterns in a prey-predator system with parental care for predators, Applied Mathematics and Computation. 237 (2014) 176189.

6. W.D. Wang, Y. Takeuchi, Y. Saito, S. Nakaoka, Prey-predator system with parental care for predators, J. Theor. Biol. 241 (2006) 451-458. 
7. J. Roughgarden, Y. Iwasa, C. Baxter, Demographic theory for an open marine population with space-limits, Ecology 66 (1985) 54-67

8. Canrong Tian, Lai Zhang, Hopf bifurcation analysis in a diffusive food-chain model with time delay, Computers and Mathematics with Applications. 66(2013)2139-2153.

9. S. Sen, P. Ghosh, S.S. Riaz, D.S. Ray, Time-delay-induced instabilities in reactiondiffusion systems, Phys. Rev. E 80 (2009) 046212.

10. S.S. Lee, E.A. Gaffney, N.A.M. Monk, The Influence of Gene Expression Time Delays on Gierer-Meinhardt Pattern Formation Systems, Bull. Math. Biol. 72 (2010) 21392160.

11. C.R. Tian, Delay-driven spatial patterns in a plankton allelopathic system, Chaos 22 (2010) 013129.

12. J. Cui, L. Chen, W. Wang, The effect of dispersal on population growth with stagestructure, Comput. Math. Appl. 39 (2000) 91-102

13. X.Y. Song, L.S. Chen, Optimal harvesting and stability for a predator-prey system with stage structure, Acta Math. Appl. Sin. 18 (2002) 423-430.

14. W.D. Wang, L.S. Chen, A predator-prey system with stage structure for predator, Comput. Math. Appl. 33 (1997) 83-91.

15. R. Kohn, Energy-driven pattern formation, in: Proceedings of the International Congress of Mathematicians, European Mathematical Society, Madrid, 2007, pp. 1-25 